\title{
The cyclin-dependent kinase inhibitor flavopiridol induces apoptosis in human leukemia cells (U937) through the mitochondrial rather than the receptor-mediated pathway
}

\author{
RH Decker ${ }^{1,2}$, Y Dai ${ }^{1}$ and S Grant ${ }^{\star, 1,2,3}$ \\ ${ }^{1}$ Department of Medicine, Medical College of Virginia, Richmond, Virginia, VA \\ 23298, USA \\ 2 Department of Biochemistry, Medical College of Virginia, Richmond, Virginia, \\ VA 23298, USA \\ ${ }^{3}$ Department of Pharmacology, Medical College of Virginia, Richmond, Virginia, \\ VA 23298, USA \\ * Corresponding author: S Grant, Department of Medicine, Medical College of \\ Virginia, MCV Station Box 230, Richmond, VA 23298. \\ Tel: (804) 828-5211; Fax (804) 828-8079
}

Received 25.9.00; revised 18.12.00; accepted 30.1.01

Edited by CJ Thiele

\begin{abstract}
Flavopiridol (FP), an inhibitor of cyclin dependent kinases 1, 2 and 4, potently induced apoptosis in U937 human monoblastic leukemia cells. This process was accompanied by characteristic morphological changes, inner mitochondrial membrane permeability transition, release of cytochrome $c$, processing of procaspases, and generation of reactive oxygen species. Significantly, the general caspase inhibitor Boc-FMK did not block the release of cytochrome $c$, whereas it did block cleavage of BID and the loss of $\Delta \Psi_{\mathrm{m}}$. Neither FP-induced apoptosis nor cytochrome $c$ release was inhibited by the pharmacological caspase-8 inhibitor IETD-FMK or endogenous expression of viral caspase-8 inhibitor CrmA. Finally, FPmediated apoptosis, but not cytochrome $c$ release, was partially blocked by the free radical scavenger LNAC. Collectively, these findings indicate that FP induces apoptosis in U937 cells via the release of cytochrome $c$ from the mitochondria and independently of activation of procaspase8. Cell Death and Differentiation (2001) 8, 715-724.
\end{abstract}

Keywords: flavopiridol; apoptosis; leukemia; caspase; cytochrome $c$

Abbreviations: FP, flavopiridol; LNAC, N-acetyl-L-cysteine; MPT, mitochondrial permeability transition; Boc-FMK, BOC-Asp(OMe)fluoromethyl ketone; IETD-FMK, Ac-Ile-Glu-Thr-Asp-fluoromethyl ketone; DEVD-FMK, Ac-Asp(OMe)-Glu(OMe)-Val-Asp(OMe)fluoromethyl ketone; PARP, poly(ADP-ribose) polymerase; TNF, Tumor Necrosis Factor; CHX, cycloheximide; FADD, Fasassociated death domain; ROS, reactive oxygen species; DMSO, dimethyl sulfoxide; ara-C, 1-D-arabinofuranosylcytosine

\section{Introduction}

Apoptosis is a regulated, energy-dependent process in which cells die in response to growth factor withdrawal, ${ }^{1}$ ligand binding, ${ }^{2}$ or cytotoxic stimuli such as drug treatment. ${ }^{3}$ It occurs in two phases, i.e., an initiation phase which may be stimulus dependent, and a common downstream effector phase which involves chro matin condensation, DNA fragmentation, and cell membrane alterations. The sequential cleavage and activation of caspases, a family of cysteine proteases, is a requisite step in both phases of this signaling cascade. Initiator caspases containing long pro-domains, including caspase-8 in CD95- (Fas/Apo-1) or TNF-induced apoptosis, ${ }^{2}$ or caspase- 9 which is triggered in response to various chemical stimuli, ${ }^{4}$ directly or indirectly activate effector caspases-3, -6 , and -7 . The latter cleave a variety of intracellular targets responsible for downstream functional and morphologic changes. ${ }^{5-7}$ Procaspase- 8 is activated at the cell membrane by receptor binding and is capable of cleaving and activating all downstream caspases ${ }^{8}$ as well as inducing release of cytochrome $c$ from the mitochondria through the cleavage of BID. ${ }^{9,10}$ In contrast, procaspase- 9 is activated downstream of drug-induced mitochondrial injury and forms a complex with cytosolic cytochrome $c$, dATP and Apaf-1, referred to as the apoptosome. ${ }^{11}$ The apoptosome, following formation of an oligomerized complex, ${ }^{12}$ cleaves and activates procaspase-3. Inasmuch as caspase-3 ultimately activates both procaspases- 8 and $-9,{ }^{13}$ both the extrinsic (i.e., receptor-mediated) and the intrinsic (i.e., mitochondrial-dependent) pathways ultimately result in pancaspase activation which may provide the cell with an amplification mechanism to ensure its demise.

Flavopiridol (NSC 649890)(FP) is a semisynthetic flavonoid that binds to the ATP-binding pocket of multiple cyclin-dependent kinases, ${ }^{14}$ proteins intimately involved in the regulation of cell cycle progression. ${ }^{15}$ It is a potent inhibitor of cyclin-dependent kinases 1,2 , and 4 in vivo, ${ }^{14,16}$ and effectively induces apoptosis in human leukemia cell lines. ${ }^{17-19} \mathrm{FP}$ is presently under investigation in phase II clinical trials. ${ }^{20}$ It is currently unclear whether the apoptotic response to FP represents a direct result of cdk inhibition ${ }^{21}$ or, alternately, a response to down-regulation of cell cycle ${ }^{22}$ or anti-apoptotic proteins. ${ }^{19,23}$ In view of early reports suggesting that FP-induced cell death may occur in the presence of $\mathrm{Bcl}-2,{ }^{19}$ and evidence that receptor-mediated cell death is relatively insensitive to suppression by this protein, ${ }^{24}$ questions have arisen regarding the dependence of FP-mediated lethality on early mitochondrial events. In this regard, a recent report has demonstrated that FP. induced apoptosis in a cervical carcinoma cell line was not attenuated by $\mathrm{Bcl}-2$ over-expression, but was blocked by inhibition of caspase $8 .^{25}$ Further, an additional study has 
demonstrated that FP treatment blocked cytochrome $c$ release in embryonal cortical neurons exposed to camptothecin. ${ }^{26}$ Taken together, these findings appear to argue against a mitochondrial-based mechanism of FP lethality.

Currently, relatively little information is available concerning early events involved in FP-mediated apoptosis, particularly in malignant hematopoietic cells. The purpose of this study was to investigate the relationship between FP, administered at clinically relevant concentrations, and mitochondrial injury, caspase activation, and cell death in human leukemia cells (U937). Our studies indicate that FP induces early cytochrome $c$ release which is not blocked by pharmacological inhibition of caspases, whereas cleavage of BID and mitochondrial membrane potential changes in response to FP are caspase-dependent. Moreover, in contrast to receptor-mediated apoptosis induced by TNF, FP-induced caspase-3 activity and apoptosis are not attenuated by inhibitors of caspase-8. Lastly, our results demonstrate that reactive oxygen species are generated in response to $\mathrm{FP}$, and that a free radical scavenger $(\mathrm{N}$ Acetyl-L-cysteine, LNAC) can attenuate the caspasemediated morphological changes downstream of cytochrome $c$ release. Collectively, these findings strongly

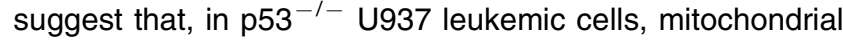
release of cytochrome $c$ and subsequent activation of caspase-9, rather than cleavage/activation of procaspase-8 and BID, are primarily responsible for initiating the apoptotic cascade in response to FP.

\section{Results}

\section{FP induces apoptosis in U937 cells}

To characterize the dose response of U937 cells to FP. mediated apoptosis, cells were exposed to a range of FP concentrations (e.g., 50-400 nM) for $18 \mathrm{~h}$ and cytocentrifuge preparations were scored for characteristic apoptotic morphologic features (i.e., nuclear condensation and fragmentation, membrane blebbing). As shown in Figure $1 \mathrm{~A}$, concentrations of FP $100 \mathrm{nM}$ induced significant apoptosis in U937 human leukemia cells. In addition, cells exposed to $200 \mathrm{nM}$ FP were lysed at $3 \mathrm{~h}$ intervals and subjected to Western analysis (Figure 1B). Caspase substrate poly (ADPribose) polymerase (PARP) was initially cleaved to an $85 \mathrm{kD}$ fragment at $6 \mathrm{~h}$, and this effect was significantly more pronounced by $12 \mathrm{~h}$.

To investigate cell cycle-related effects of FP, cells were exposed to $100-300 \mathrm{nM}$ FP in the presence or absence of pan-caspase inhibitor Boc-FMK, fixed, and analyzed by flow cytometry for DNA content. In the absence of BocFMK (Figure 2A), a clear increase in the subdiploid population was noted with increasing FP concentration, accompanied by pronounced decreases in the $S$ and $G_{2} / M$ phases and a moderate decrease in the $G_{1}$ population. However, the addition of Boc-FMK (Figure 2B) effectively blocked FP-mediated increases in the subdiploid fraction, thereby demonstrating the caspase-dependency of FP. mediated DNA fragmentation. Under these conditions, the $\mathrm{G}_{0} / \mathrm{G}_{1}$ population was unperturbed, whereas the S-phase
A.

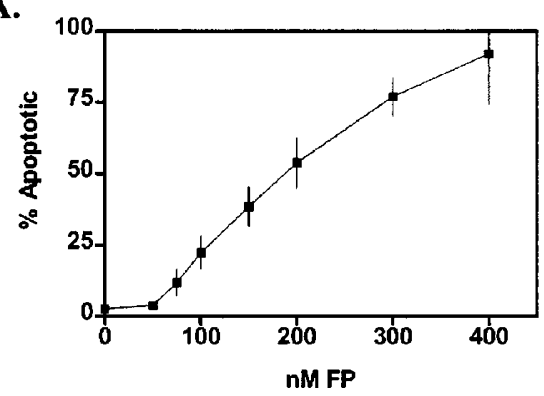

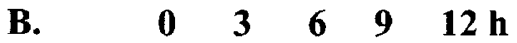

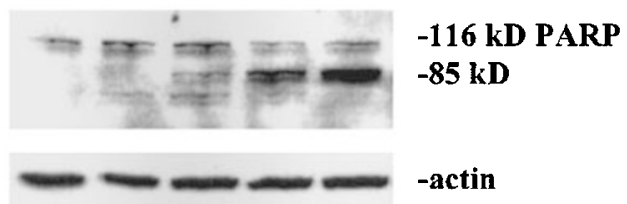

Figure 1 FP induces apoptosis in U937 cells. (A) U937 cells were treated with FP indicated concentrations for $18 \mathrm{~h}$, cytocentrifuged, stained with modified Wright-Giemsa, and assessed for the characteristic morphological changes of apoptosis. The data represent five independent experiments (S.E.M.). (B) In a parallel experiment, cells treated with $200 \mathrm{nM}$ FP were lysed at 3-h intervals and subjected to electrophoresis and Western analysis for PARP

A. $\quad$ FP

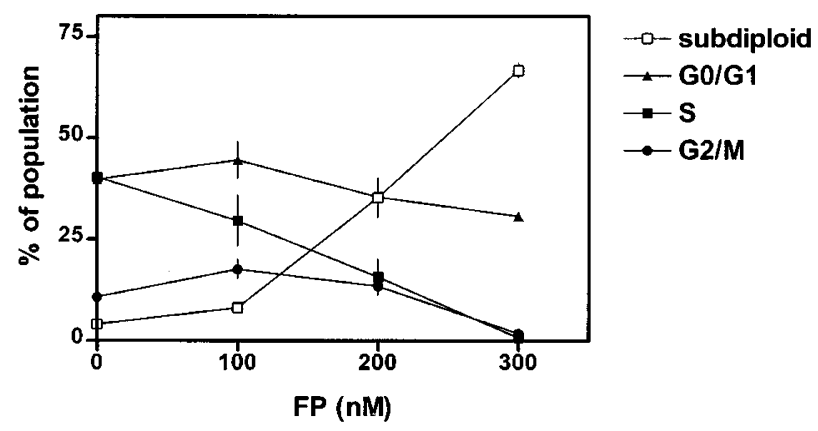

B.

$F P+B o c-f m k$

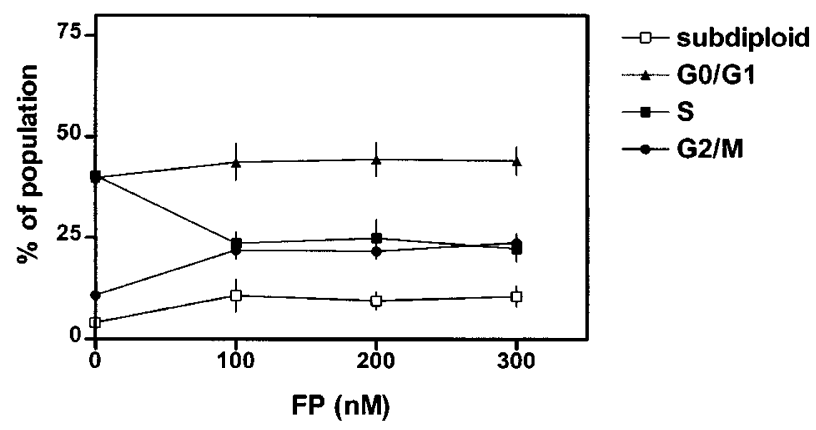

Figure 2 Inhibition of FP-mediated apoptosis results in an increase in the G2/ $\mathrm{M}$ population. U937 cells were treated with FP for $24 \mathrm{~h}$ either alone $(\mathbf{A})$ or following treatment with the broad specificity caspase inhibitor Boc-FMK (B). Treated cells were fixed, stained with propidium iodide, and subjected to flow cytometric analysis for DNA content. The graphs represent changes in cell cycle populations determined after three independent experiments (S.E.M.) 
and $G_{2} M$ fractions displayed a significant decrease and increase, respectively $(P<0.05$ versus untreated control cells in each case). These findings are consistent with past reports demonstrating that $F P$ induces $G_{2} / M$ phase arrest. ${ }^{27}$

\section{FP treatment induces release of cytochrome $c$, sequential cleavage of caspases, and loss of mitochondrial membrane potential}

In order to determine whether FP treatment activates the mitochondrial cell death pathway, U937 cells were exposed to 200 nM FP for 8 h. At 1 h intervals, samples were lysed with digitonin and the resulting cytoplasmic fractions subjected to Western analysis (Figure $3 \mathrm{~A})$. Etoposide (100 M, $3 \mathrm{~h}$ ) was used as a positive control for cytochrome $c$ release and caspase activation. In addition, cells were pretreated for

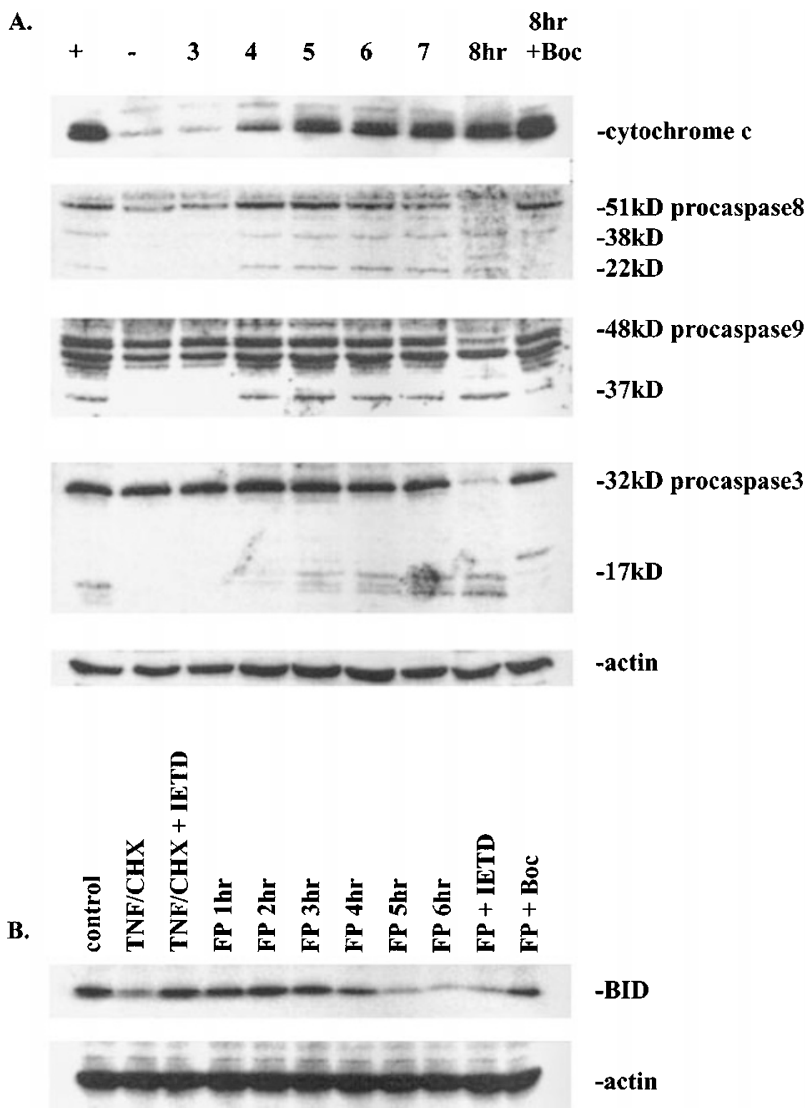

Figure 3 FP induces a time-dependent release of cytochrome $c$ from the mitochondria and cleavage of procaspases. (A) Cells were treated with $200 \mathrm{nM}$ FP for the indicated times and the cytosolic fraction subjected to Western analysis for the appearance of cytochrome $c$ (top panel); whole cell lysates from the same experiment were assessed for cleavage of procaspases (bottom 4 panels). Etoposide-treated U937 cells $(100 \mathrm{M}, 3 \mathrm{~h})$ were used as a positive control for cytochrome $c$ release and caspase cleavage $(+)$. In parallel, cells were pre-treated for $30 \mathrm{~min}$ with broad-specificity-caspase inhibitor Boc-FMK (20 M) followed by $200 \mathrm{nM} \mathrm{FP}$ for $8 \mathrm{~h}(8 \mathrm{~h}+\mathrm{Boc})$. The results shown are representative of three experiments. (B) Cells treated FP for 1-6h were subjected to Western analysis to determine the chronology of BID cleavage. TNF $(10 \mathrm{ng} / \mathrm{ml}, 3 \mathrm{~h})$ in the presence of cycloheximide $(1 \mathrm{M})(\mathrm{TNF} /$ $\mathrm{CHX})$, alone or in the presence of caspase-8 inhibitor IETD-FMK (20 M) was used as a positive control. FP in the presence of IETD-FMK or Boc-FMK was also examined as shown
30 min with the broad-specificity caspase inhibitor Boc-FMK, after which they were exposed to $200 \mathrm{nM}$ FP for $8 \mathrm{~h}$ to evaluate the role of caspase activation in these steps. Cytochrome $c$ was first detected in the cytoplasmic fraction $4 \mathrm{~h}$ after treatment with FP. While a very small amount of protein was evident at $3 \mathrm{~h}$, it was equivalent to the amount seen in the negative control, and presumably represents mitochondrial contamination of the cytosolic fraction. ${ }^{28}$ Coincident with release of cytochrome $c$ from the mitochondrial intermembrane space, the active 38 and $22 \mathrm{kD}$ fragments of procaspase- 8 , the active $37 \mathrm{kD}$ fragment of procaspase- 9 , and the active $17 \mathrm{kD}$ fragment of procaspase3 became faintly discernible. This is consistent with reports that release of cytochrome $\mathrm{c}$ is rapidly followed by procaspase activation. $^{29}$ However, it is important to note that whereas Boc-FMK pre-treatment substantially reduced cleavage of procaspases-8, -9 , and -3 , it did not attenuate cytochrome $c$ release, providing evidence that the latter represents a primary event rather than a consequence of procaspase- 8 activation.

In parallel experiments, cells exposed to FP were subjected to Western analysis to examine the temporal pattern of BID cleavage (Figure 3B). BID is a known substrate of caspases- 8 and -3 , cleavage of which is required for release of cytochrome $c$ in response to receptor-mediated programmed cell death. ${ }^{30,31}$ TNF in the presence of cycloheximide (TNF/CHX), a well-described stimulus for receptor-mediated killing in U937 cells, was used as a positive control for caspase-8-dependent BID cleavage and subsequent cytochrome $c$ release. ${ }^{13}$ BID cleavage was evident after treatment with TNF, and was blocked by specific caspase-8 inhibitor IETD-FMK, as anticipated. However, FP-induced BID cleavage was not attenuated by IETD-FMK but was blocked by the general caspase inhibitor Boc-FMK. Together, these findings indicate that, unlike TNF/CHX, FP-mediated BID cleavage is not solely dependent upon caspase- 8 activity, but occurs as a result of general caspase activation downstream of cytochrome $c$ release.

\section{Loss of inner mitochondrial membrane potential lies downstream of caspase activation in FP-treated cells}

To investigate the relationship between the mitochondrial permeability transition (MPT) and cytochrome $c$ release, FPtreated U937 cells were stained with cationic mitochondrial dye $\mathrm{DiOC}_{6}(3)$ and analyzed by flow cytometry. Cells in which the mitochondrial inner membranes have depolarized exhibit low fluorescence (Figure 4). Loss of membrane potential $\left(\Delta \Psi_{\mathrm{m}}\right)$ did not precede cytochrome $c$ release. Moreover, in marked contrast to results obtained for cytochrome $c$ release, caspase inhibition by Boc-FMK substantially blocked the reduction in $\Delta \Psi_{\mathrm{m}}$, indicating that this event is secondary to caspase activation rather than an initiating stimulus for redistribution of cytochrome $c$. Identical results were obtained when cells were stained with the lipophilic cationic dye JC1, which loses aggregation-dependent red fluorescence and increases monomeric green fluorescence upon mitochondrial depolarization (data not shown). 


\section{FP-mediated apoptosis is not dependent upon the activity of caspase-8}

To investigate whether cell death in response to FP was dependent upon activation of caspase-8, U937 cells were exposed to FP $(200 \mathrm{nM})$ in the presence of specific caspase8 inhibitor IETD-FMK, specific caspase 3 inhibitor DEVDFMK, or the broad specificity caspase inhibitor Boc-FMK. After $12 \mathrm{~h}$, cell lysates were assayed for cleavage of substrates of caspase 8 (IETD-NA) and caspase 3 (DEVD$\mathrm{NA}$ ) to their colorimetric product (Figure $5 \mathrm{~A}$ and $\mathrm{B}$ ). TNF in the presence of cycloheximide was used as a positive control for extrinsic caspase activation (i.e., caspase 8-dependent caspase 3 activation); etoposide (VP16, $100 \mathrm{M}, 4 \mathrm{~h}$ ) was included as an activator of the intrinsic, or mitochondrial, apoptotic pathway. ${ }^{13}$ Pretreatment with IETD-FMK blocked caspase 8 activity induced by all treatments, but blocked caspase 3 activity only in response to TNF/CHX. FP treatment induced an approximately 13-fold induction of caspase 3 activity which was not significantly attenuated in the presence of IETD-FMK, indicating that FP-mediated caspase 3 activity is not dependent upon activation of caspase 8.

In parallel experiments, cytocentrifuge preparations were scored for morphologic apoptosis (Figure 6A) and analyzed by flow cytometry for loss of $\Delta \Psi_{\mathrm{m}}$ (Figure 6B) following $18 \mathrm{~h}$ of FP exposure $(200 \mathrm{nM})$ in the presence of caspase inhibitors. While Boc-FMK and DEVD-FMK blocked apoptosis in response to FP, IETD-FMK had no effect. In contrast, apoptosis in response to TNF/CHX was blocked equally by IETD-FMK, DEVD-FMK or Boc-FMK. Etoposide (VP16) induced apoptosis which was blocked by Boc-FMK and DEVD-FMK but not by IETD-FMK, as anticipated. The loss of $\Delta \Psi_{\mathrm{m}}$ in response to FP was attenuated after IETDFMK treatment, although not to the extent observed in cells subjected to TNF/CHX treatment. This effect was, however, similar to that observed in VP16-treated cells, and suggests that induction of the MPT by FP represents a downstream event that is dependent in part upon the actions of caspases, including caspases- 6 and -8 .

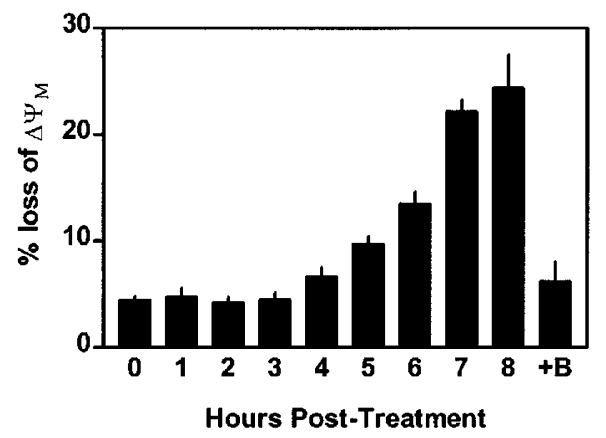

Figure 4 FP induces loss of mitochondrial transmembrane potential which is dependent upon caspase activation. Cells treated with $200 \mathrm{nM}$ FP for $1-8 \mathrm{~h}$ were incubated with $40 \mathrm{nM} \mathrm{DiOC}{ }_{6}(3)$ for $15 \mathrm{~min}$, and inner mitochondria membrane potential assessed by flow cytometry. In parallel, cells pretreated with pan-caspase inhibitor Boc-FMK were exposed to $200 \mathrm{nM}$ FP for $8 \mathrm{~h}$. The mean number of cells exhibiting low mitochondrial membrane potential (S.E.M.) for three independent experiments was plotted
To compare the roles of caspase activation in FPmediated versus receptor-mediated cell death further, U937 cells treated with FP, TNF/CHX, or VP16 in the presence or absence of Boc-FMK were selectively lysed with digitonin and subjected to Western analysis for cytosolic cytochrome $c$ (Figure 7). Cytochrome $c$ release in response to TNF/ $\mathrm{CHX}$ was blocked by caspase inhibition, reflecting dependence upon receptor-mediated caspase-8 activity. In contrast, FP-mediated cytochrome $c$ release occurred in the presence of Boc-FMK as noted previously, supporting the concept that this event is caspase-independent. Similarly, VP16-induced cytochrome $c$ release occurred despite caspase inhibition. In all cases, the appearance of active fragments of procaspase- 3 was also inhibited by Boc-FMK (data not shown).

In order to verify caspase-8-independence, U937 cells were stably transfected with a construct that constitutively expresses CrmA, a cowpox virus-encoded inhibitor of caspases-8 and $-1 .^{32}$ Two clonally selected populations of CrmA-expressing cells (designated NE3 and NF7) exhibited marked resistance to TNF/CHX, VP16, and FP-induced caspase 8 activation in comparison to both empty vector

A.
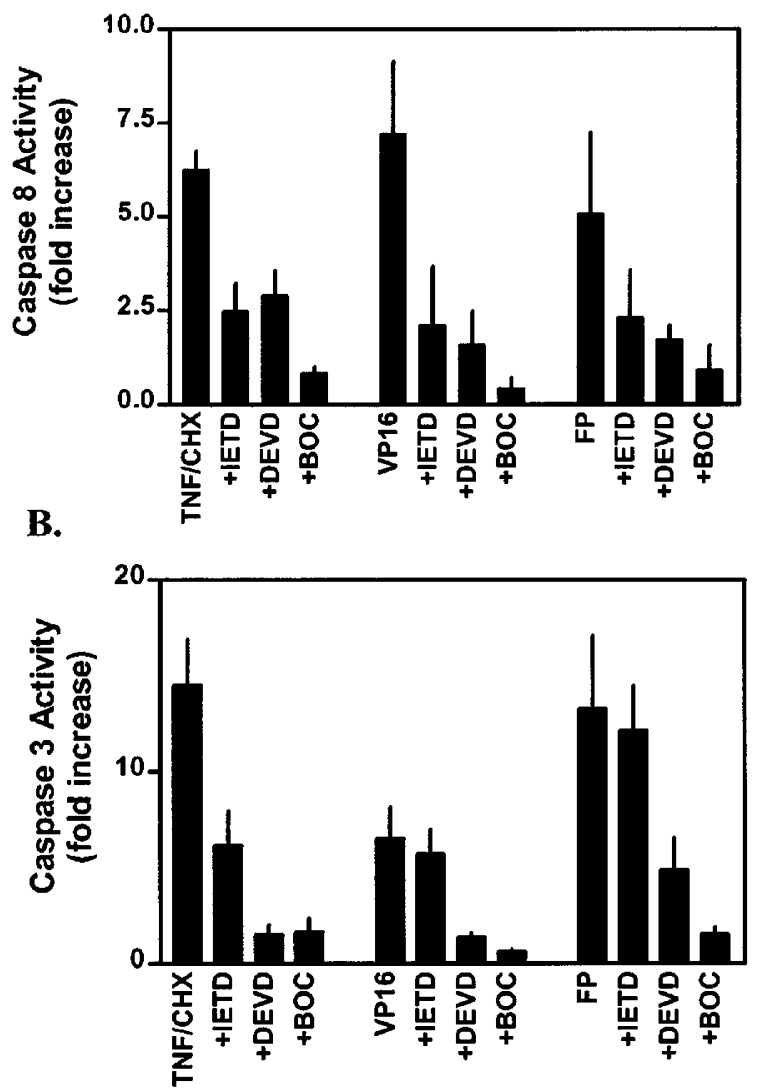

Figure 5 A specific inhibitor of caspase 8 blocks activation of caspase 3 following treatment with TNF but not following VP16 or FP. Cells treated with TNF (10 ng/ml, $4 \mathrm{~h}$ ) in the presence of Cycloheximide (CHX), etoposide $(100 \mathrm{M}$, $4 \mathrm{~h})(\mathrm{VP} 16)$ or FP $(200 \mathrm{nM}, 12 \mathrm{~h}$ ) were lysed assayed for activity of caspase 8 (A) and caspase 3 (B). The results of three independent experiments are shown 
controls (pcDNA3.1) and parental U937 cells (Figure 8A). In TNF/CHX-treated CrmA cells, but not in FP- or VP16treated cells, caspase 3 activation was also attenuated by CrmA (Figure 8B) in a manner similar to that observed in cells pre-treated with IETD-FMK (Figure 5). Cytocentrifuge preparations from these same experiments demonstrated that $\mathrm{CrmA}$-expressing cell lines were significantly resistant to TNF/CHX-mediated apoptosis compared to empty vector controls and to parental U937 cells (Figure 9A) but were sensitive to VP16, as anticipated. FP induced apoptosis in CrmA expressing cells to the same extent as in vector controls and untransfected U937 cells, indicating that caspase-8 activity is not required for the cellular response to FP. FP- and VP16-induced MPT was attenuated in a CrmA-expressing cell line (Figure 9B), similar to results obtained in IETD-FMK pretreated cells. Together, these findings argue against a major role for receptor pathwaymediated caspase activation in FP-induced cell death in U937 cells.

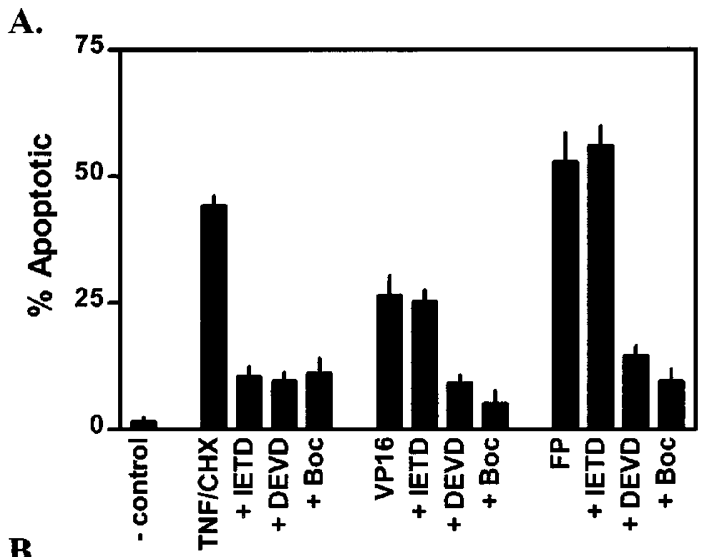

B.

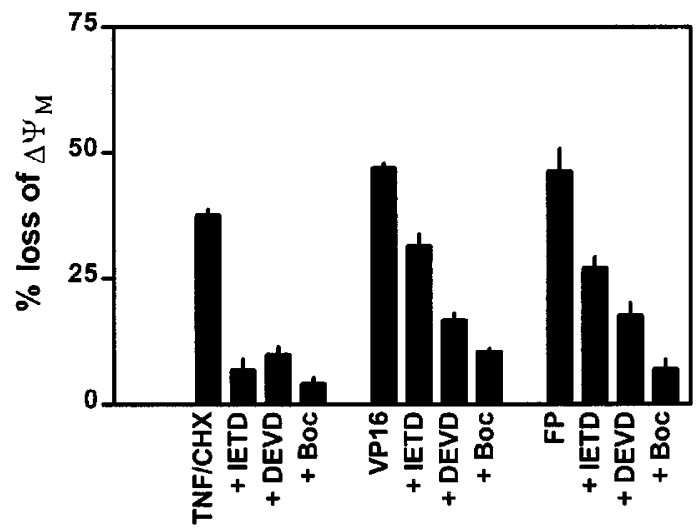

Figure 6 FP-mediated apoptosis and loss of mitochondrial membrane potential is not dependent upon the activity of caspase-8. U937 cells were pretreated with specific caspase-8 inhibitor IETD-FMK, specific caspase 3 inhibitor DEVD-FMK, or with general caspase inhibitor Boc-FMK, exposed to $200 \mathrm{nM} \mathrm{FP}$ for $18 \mathrm{~h}$, and assessed for morphological apoptosis (A) and mitochondrial membrane potential by $\mathrm{DiOC}_{6}(3)$ retention $(\mathbf{B})$. Treatment with TNF- $(10 \mathrm{ng} / \mathrm{ml}, 4 \mathrm{~h})$ in the presence of cycloheximide (TNF/CHX) was used as a control for receptor-mediated apoptosis, etoposide (100M, 4h)(VP16) served as a control for caspase-8-independent cell death

\section{FP-mediated apoptosis is dependent upon generation of radical oxygen species}

To determine whether exposure to FP resulted in the generation of oxygen radicals, treated cells (200 nM FP, $6 \mathrm{~h}$ ) were loaded with CDCFDA, a fluorogenic acetoxymethyl ester diacetate, and incubated for $2 \mathrm{~h}$ at $37^{\circ} \mathrm{C}$. The dye is deesterified within cells and fluoresces upon oxidation by peroxide radicals. ${ }^{33}$ FP-treated samples were analyzed by flow cytometry and compared to untreated controls; a representative experiment (Figure 10A) demonstrates that FP treatment results in a significant increase in cellular peroxide radicals.

To investigate the role of oxygen radical generation in the apoptotic response of U937 cells to FP, cells were

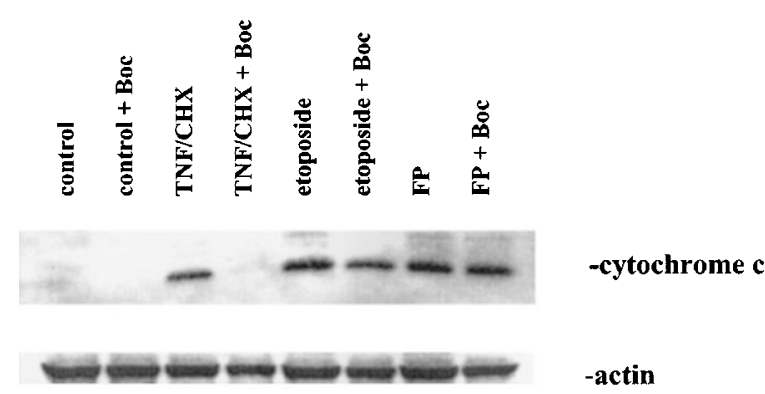

Figure 7 Caspase inhibition does not block FP-induced cytochrome $c$ release. Cytosolic fractions of cells treated with TNF/CHX $(10 \mathrm{ng} / \mathrm{ml} \mathrm{TNF}, 1 \mathrm{M}$ $\mathrm{CHX}, 3 \mathrm{~h}), \mathrm{VP} 16(100 \mathrm{M}, 3 \mathrm{~h})$, or FP $(200 \mathrm{nM}, 6 \mathrm{~h})$ in the absence or presence of Boc-FMK (20 M, 30 min pretreatment) were subjected to Western analysis for the appearance of cytochrome $c$
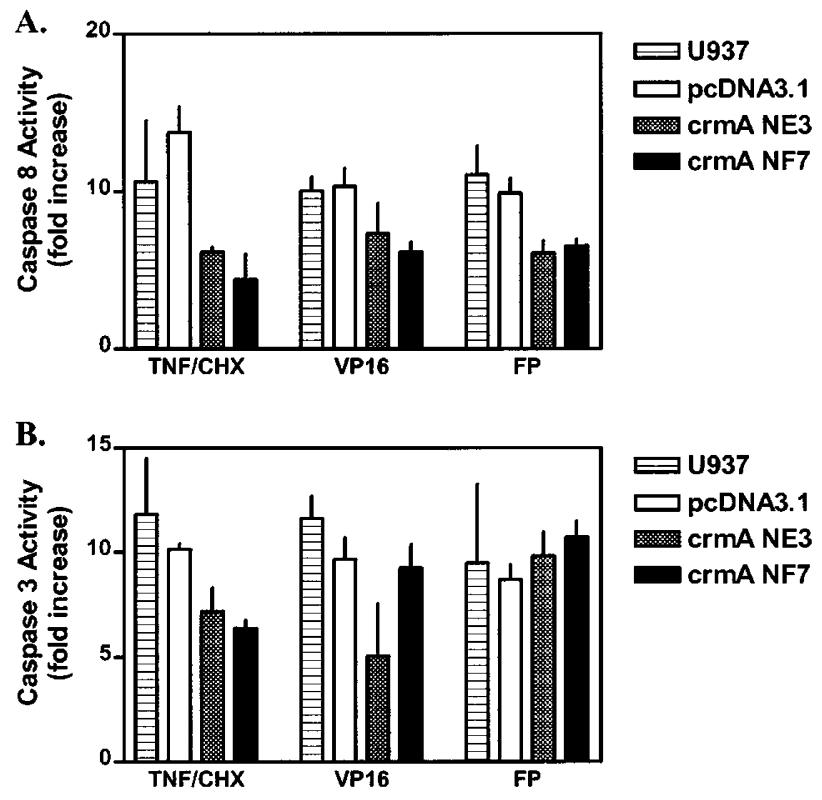

Figure 8 CrmA-expressing U937 are resistant to FP-mediated activation of caspase 8, but not that of caspase 3 . U937 cells, two clonal populations of crmA-expressing cells (NE3, NF7) and a vector control cell line (pcDNA3.1) were expose to TNF $(10 \mathrm{ng} / \mathrm{ml}, 4 \mathrm{~h})$ in the presence of CHX, VP16 (100 M, $4 \mathrm{~h})$, or FP $(200 \mathrm{nM}, 12 \mathrm{~h})$ and lysated assayed for activity of caspase $8(\mathbf{A})$ and caspase 3 (B). The results of three independent experiments are shown 

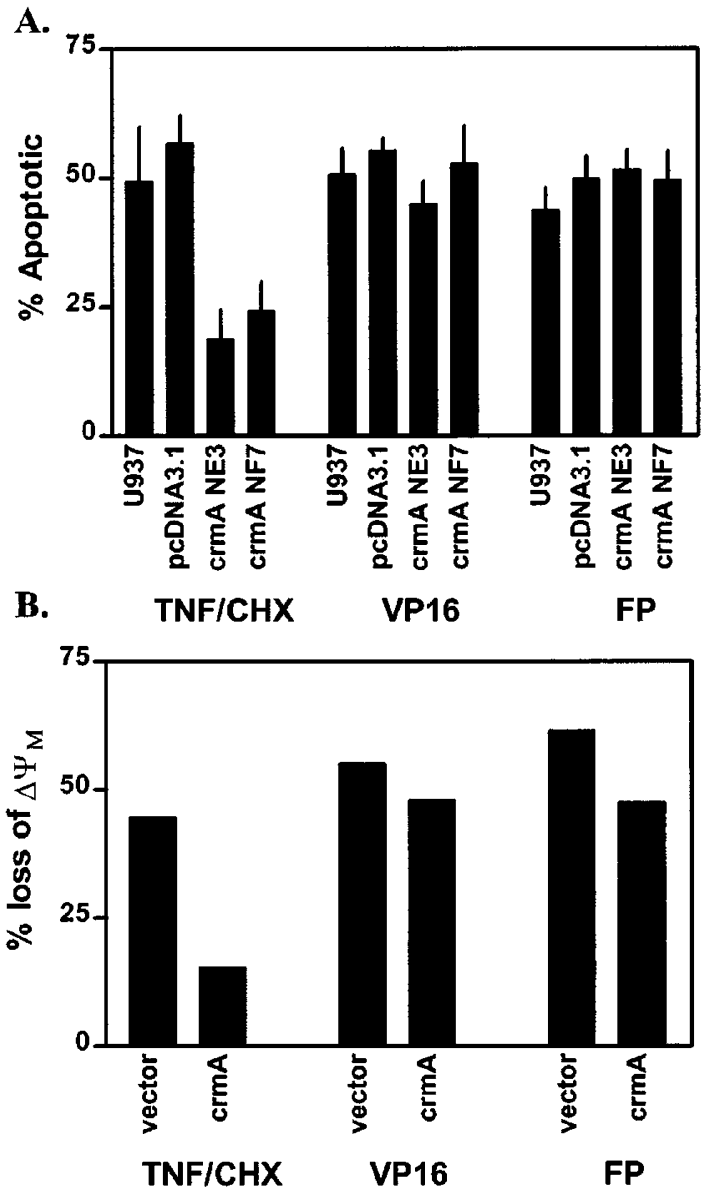

Figure 9 CrmA-expressing U937 cells are sensitive to FP-mediated apoptosis and MPT. U937 cells stably transfected with a plasmid expressing viral caspase inhibitor CrmA (NE3 and NF7) were exposed to TNF/CHX, VP16, or FP in parallel with empty vector controls (pcDNA3.1) and parental U937 cells and examined for morphologic apoptosis $(\mathbf{A})$. The data represent five independent experiments. Selected cell lines were analyzed for mitochondrial membrane potential change by loss of $\mathrm{DiOC}_{6}(3)(\mathrm{B})$. This is representative of five experiments

exposed to $200 \mathrm{nM} F P$ following $1 \mathrm{~h}$ pre-treatment with the GSH precursor N-Acetyl-L-cysteine (LNAC, $10 \mathrm{mM}$ ). Cytocentrifuge preparations of treated cells were scored for morphologic evidence of apoptosis (Figure 10B), and additionally lysed with digitonin for Western analysis of cytosolic cytochrome $c$ (Figure 10C). LNAC pre-treatment significantly $(P<0.05)$ blocked the characteristic morphologic features of apoptosis, although the effect was incomplete. UV irradiation was used as a positive control for oxygen-radical-dependent apoptosis and was also only partially blocked by LNAC pre-treatment. Lower concentrations of LNAC blocked the apoptotic response to a lesser extent (data not shown), whereas increasing concentrations induced necrosis in U937 cells and therefore were not evaluated. Western analysis of the cytosolic fraction revealed that FP-mediated cytochrome $c$ release was not inhibited by LNAC when the latter was administered at a concentration that attenuated apoptosis, and suggests that free radical actions lie downstream of this event.

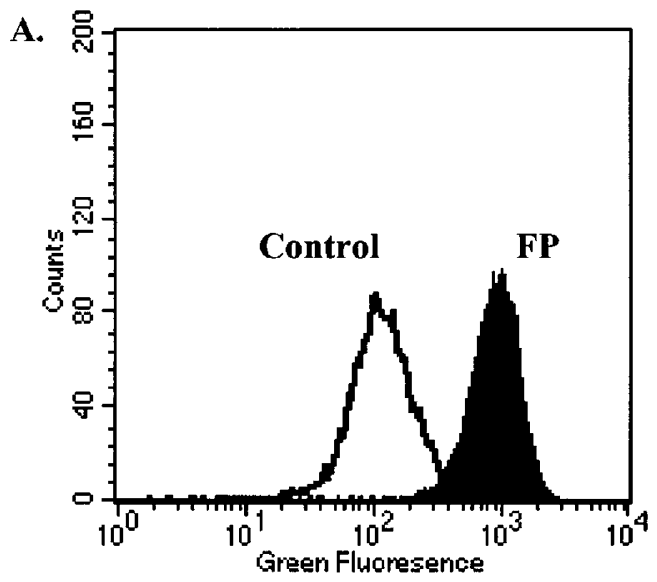

B.
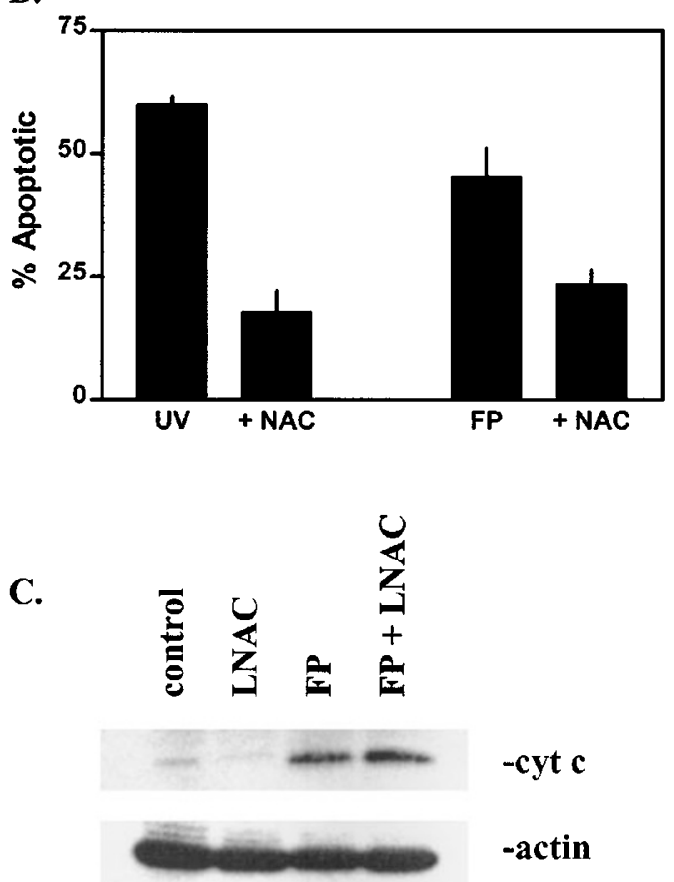

Figure 10 FP-mediated apoptosis is dependent upon generation of radical oxygen species. (A) U937 cells were treated with FP $(200 \mathrm{nM}, 6 \mathrm{~h})$ and then stained with CDCFDA $\left(500 \mathrm{nM}, 2 \mathrm{~h}, 37^{\circ} \mathrm{C}\right)$, which fluoresces upon intracellular oxidation by peroxide radicals. Samples were analyzed by flow cytometry in comparison with untreated controls. This is representative of three experiments. (B) Cells were pre-treated with oxygen radical scavenger $\mathrm{N}$ acetyl-L-cysteine (NAC) for $1 \mathrm{~h}$, exposed to FP $(200 \mathrm{nM}, 8 \mathrm{~h})$, and scored for morphologic apoptosis. UV irradiation ( $15 \mathrm{~min}$ ) was used as a positive control for peroxide radical generation-dependent cell death. (C) Cytosolic fractions were subject to Western analysis for cytochrome $c$ release from mitochondria

\section{Discussion}

The findings presented herein demonstrate that the CDK inhibitor FP, when administered at clinically achievable concentrations, potently induces mitochondrial damage in U937 human leukemia cells, leading in turn to caspase activation and initiation of the apoptotic cascade. CDK inhibitors, which include, in addition to $\mathrm{FP}$, roscovitine, olomoucine, butyrolactone, and the paullones, are currently 
the subject of considerable interest as antineoplastic agents. ${ }^{21}$ This interest has been stimulated by the discovery that in addition to their capacity to overcome the growth dysregulation characteristic of neoplastic cells, CDK inhibitors are also potent inducers of apoptosis. ${ }^{34}$ In view of abundant evidence that disruption of the cell cycle is one of the most effective inducers of cell death, ${ }^{35}$ it is presumed that CDK inhibitor-induced apoptosis stems from interference with cell cycle traverse. However, the early events surrounding initiation of apoptosis by FP or other CDK inhibitors remain largely unexplored.

To date, three major pathways of apoptosis have been described. The first, referred to as the intrinsic pathway, involves mitochondrial damage, such as loss of the mitochondrial membrane potential $\left(\Delta \Psi_{\mathrm{m}}\right)$ and/or release of pro-apoptogenic proteins from the inner mitochondrial space, including cytochrome $c$ or AIF (apoptosis-inducing factor). ${ }^{36,29}$ These events, in turn, lead to activation of initiator caspases, particularly procaspase-9, which triggers cleavage and activation of effector caspases directly responsible for cellular disassembly such as procaspases-3, and-7. ${ }^{6}$ Alternatively, binding of certain ligands to their cell surface receptors (e.g., Fas-L, Apo, CD95) triggers a cascade of events culminating in activation of FADD (fas-associated death domain) and cleavage/ activation of procaspase-8. ${ }^{37,38}$ This cascade, referred to as the extrinsic pathway, is primarily involved in apoptosis associated with the TNF family of cytokines, ${ }^{39,40}$ and may proceed independently of mitochondrial damage. Nevertheless, receptor pathway-associated activation of procaspase- 8 can lead to cleavage and activation of BID, which is capable of inducing cytochrome $c$ release as a secondary event. ${ }^{9}$ A third pathway of apoptosis involves members of the granzyme $B$ family. ${ }^{41}$ In the case of most chemotherapeutic agents, apoptosis has been linked to mitochondrial damage, including loss of the $\Delta \Psi_{\mathrm{m}}$ or release of cytochrome $c$ into the cytoplasm. ${ }^{4}$ However, evidence exists that the extrinsic apoptotic pathway may be involved in certain forms of drug-induced cell death (e.g., that associated with doxorubicin), ${ }^{42}$ although this phenomenon appears to be highly stimulus-and cell typedependent. $^{43}$ It should be noted that drug-induced apoptosis (e.g., by agents such as VP-16) can primarily trigger mitochondrial injury, but may also induce procaspase- 8 and BID cleavage as secondary events, which in turn potentiate mitochondrial dysfunction. ${ }^{13}$ In this way, the receptor-mediated pathway can serve to amplify the apoptotic response to agents that initially act by triggering mitochondrial damage.

FP-induced apoptosis differs in several respects from that associated with most conventional cytotoxic agents, and such considerations raise the possibility that FP may activate a unique apoptotic pathway. For example, FP has been shown to induce apoptosis to an equivalent extent in $\mathrm{p5}^{+}$and $\mathrm{p53}^{-}$cells, ${ }^{27}$ and to act in the presence of $\mathrm{Bcl}-$ 2 . $^{18,19}$ Because $\mathrm{Bcl}-2$ is generally more effective in blocking apoptosis proceeding through the mitochondrial rather than the receptor-related pathway, ${ }^{24,44}$ the latter finding would be compatible with an extrinsic pathway-associated mechanism of FP lethality. In support of this concept, a recent study has demonstrated that cervical carcinoma cells undergo FP-mediated apoptosis independently of endogenous Bcl-2 over-expression, and in a caspase-8 dependent manner. ${ }^{25}$

In sharp contrast, our findings in U937 monocytic leukemia cells provide several lines of evidence arguing that clinically achievable concentrations of FP induce apoptosis by triggering release of cytochrome $c$ from the mitochondria and subsequent caspase activation, rather than through primary activation of receptor-dependent caspase cascades. While an analysis of the temporal pattern of pro-caspase cleavage was inconclusive, presumably due to the rapid nature of caspase activation following the appearance of cytochrome $c,{ }^{29}$ the results of caspase inhibition studies argued strongly that caspase-8 did not play a primary role in the cellular response to FP. First, the broad-specificity caspase inhibitor Boc-FMK blocked caspase activation and BID cleavage in response to FP, but failed to attenuate cytochrome $c$ release, indicating that this event is not caspase- or BIDdependent. Secondly, IETD-FMK significantly blocked caspase-8 activity in response to FP without attenuating caspase-3 activity, BID cleavage, or apoptosis. This was in sharp contrast to TNF/CHX treatment, in which caspase 8 inhibition blocked BID cleavage, caspase-3 activity, and the apoptotic response. Finally, U937 cells stably expressing viral caspase inhibitor CrmA, which blocked caspase8 activity, were fully susceptible to FP-mediated caspase-3 activity or apoptosis, but were highly resistant to TNFmediated death. Together, these findings suggest that the primary action of FP is to induce cytochrome $c$ release by the intrinsic or mitochondrial pathway, and that activation of components of the extrinsic pathway is a consequence, rather than a cause, of this event. As in the case of conventional cytotoxic drugs, ${ }^{13}$ the possibility that secondary activation of procaspase- 8 and BID may serve to amplify FP-mediated mitochondrial damage and apoptosis cannot be excluded. In fact, based on the evidence that BID cleavage and caspase 8 activation are observed downstream of cytochrome $c$ release and caspase 3 activity in response to $\mathrm{FP}$, this does appear to be the case.

There is some debate as to whether release of cytochrome $c$ or loss of $\Delta \Psi_{\mathrm{m}}$ represents the primary trigger for the apoptotic program. The ability of certain inhibitors of the MPT (e.g., the adenine nucleotide transporter antagonist bongkrekic acid) to block some forms of apoptosis, ${ }^{45}$ and the observation that under some circumstances, apoptosis can proceed in the absence of cytochrome $c$ release ${ }^{46}$ argues for the latter. However, numerous studies have shown that in response to various stimuli, cytochrome $c$ release precedes the loss of $\Delta \Psi_{\mathrm{m}}{ }^{47}$ and that the features of apoptosis (e.g., PARP degradation) can under some circumstances be dissociated from the loss of $\Delta \Psi_{\mathrm{m}}{ }^{29}$ In accord with the latter findings, FP-induced mitochondrial discharge and apoptosis were prevented by co-treatment with Boc-FMK, whereas cytochrome $c$ release was not. This indicates that in U937 cells the lethal actions of FP are unlikely to represent a direct consequence of loss of the $\Delta \Psi_{\mathrm{m}}$; instead, collapse of the $\Delta \Psi_{\mathrm{m}}$ may reflect, as 
previously proposed, ${ }^{48}$ disruption of respiratory chain that accompanies loss of cytochrome $c$ from the mitochondrial intermembrane space and/or caspase activation.

The role of free radical production in the apoptotic process may vary with the stimulus and cell type. In some studies, induction of apoptosis by chemotherapeutic drugs (e.g., ara-C) has been linked to production of reactive oxygen species (ROS), ${ }^{49,50}$ whereas other studies have suggested that ROS generation represents a consequence rather than a cause of cell death. ${ }^{51}$ Free radical scavengers such as LNAC or PDTC have proven useful in attempts to address this issue. In the present studies, LNAC significantly, although incompletely, blocked FPmediated apoptosis, but had no effect on cytochrome $c$ release. These findings suggest that free radical production lies downstream of FP-mediated mitochondrial injury. It remains possible, however, that free radical generation by FP could serve to amplify the apoptotic response to this agent.

In summary, the present studies demonstrate that induction of apoptosis by FP in human leukemia cells (U937) proceeds through the intrinsic, cytochrome $c$-related pathway, and is not dependent upon the extrinsic, procaspase-8-associated cascade. However, several questions concerning FP-mediated lethality remain to be answered. For example, the mechanism by which FPrelated cell cycle disruption triggers mitochondrial dysfunction, and more specifically, cytochrome $c$ release, is unclear, as is the basis for the discrepancies between the present results in human leukemia cells and those suggesting a caspase-8-dependent mechanism for FPrelated lethality in cervical carcinoma cells. ${ }^{25}$ It will also be important to determine whether the ability of FP to promote cytochrome $c$ release in leukemic cells, in contrast to its observed inhibitory effects in embryonal cortical neurons ${ }^{26}$ could serve as a possible basis for therapeutic selectivity. Finally, it will be necessary to reconcile reports that FP induces cell death in Bcl-2 over-expressing cells with evidence that $\mathrm{Bcl}-2$ acts, at least in part, by blocking mitochondrial cytochrome $c$ release. ${ }^{44}$ The reported ability of FP to down-regulate the expression of $\mathrm{Bcl}-2$ and related proteins (i.e., in primary CLL cells ${ }^{23}$ ) may bear on this issue. In this regard, the availability of U937 cells ectopically expressing $\mathrm{Bcl}-2^{52}$ should help to resolve this question. Accordingly, such studies are currently in progress.

\section{Materials and Methods}

\section{Cell lines}

U937 $7^{53}$ cells were obtained from ATCC (Rockville, MD, USA) and cultured in PRMI 1640 medium supplemented with sodium pyruvate, minimal essential medium, essential vitamins, L-glutamate, penicillin, streptomycin, and $10 \%$ heat-inactivated fetal bovine serum (Gibco). CrmA-expressing cell lines were obtained by transfecting U937 cells (Multiporator, Eppendorf) with CrmA cDNA (kindly provided by Dr Kapil Bhalla, H. Lee Moffitt Cancer Center, Tampa, FL, USA) cloned into pcDNA3.1 (Invitrogen); empty vector transfectants served as negative controls. Clonal populations were established by limiting dilution and transfectants were maintained in the presence of $400 \mathrm{~g} / \mathrm{ml}$ G418 (Life Technologies). CrmA-expressing clones were screened by Western blot with anti-CrmA antibody (Pharmingen).

\section{Drug treatments}

Ara-C and Etoposide (Sigma) were freshly prepared in sterile water. Flavopiridol, kindly provided by Dr. Edward Sausville (National Cancer Institute, Bethesda, MD, USA), was dissolved in DMSO and diluted in sterile PBS before use. Human recombinant TNF (Calbiochem), BocFMK and IETD-FMK (Enzyme Systems Products) were dissolved in DMSO. N-acetyl-L-cysteine (Calbiochem) was prepared in sterile water immediately before use. For all experiments, cells in log phase growth were suspended at $2 \times 10^{5}$ cells $/ \mathrm{ml}$ and exposed to treatment for the times indicated.

\section{Assessment of apoptosis}

Cell cultures were prepared by cytocentrifugation and stained with Diff-Quick stain set (Dade Diagnostic) and viewed by light microscopy. Experiments were performed independently in triplicate and in each case, five randomly selected fields were evaluated for characteristic apoptotic morphology (i.e., chromatin condensation and fragmentation, membrane blebbing).

\section{Cytosolic fractionation}

Cells were washed in PBS and lysed by addition of digitonin buffer ( $75 \mathrm{mM} \mathrm{NaCl}, 8 \mathrm{mM} \mathrm{Na}_{2} \mathrm{HPO}_{4} 1 \mathrm{mM} \mathrm{NaH}_{2} \mathrm{PO}_{4}, 1 \mathrm{mM}$ EDTA, $250 \mathrm{mM}$ sucrose, digitonin $35 \mathrm{~g} / 4 \times 10^{6}$ cells) for $60 \mathrm{~s}$ and centrifuged at $14000 \mathrm{~g}$ for $2 \mathrm{~min}^{54}$ The supernatants were diluted $1: 1$ with $2 \times$ SDS sample buffer and frozen at $-70^{\circ} \mathrm{C}$ for Western analysis.

\section{Western blot analysis}

Treated cells were washed in cold PBS and lysed by addition of SDS sample buffer $(60 \mathrm{mM}$ Tris, $\mathrm{pH} \mathrm{6.8,4 \%} \mathrm{sodium} \mathrm{dodecyl} \mathrm{sulfate,}$ $5.76 \mathrm{mM} \beta$-mercaptoethanol, 10\% glycerol) and briefly sonicated. Protein levels were quantified using Coomassie protein assay reagent (Pierce, Rockford, IL, USA.) Extracts were boiled for $10 \mathrm{~min}$, and $25 \mathrm{~g}$ total protein was fractioned by SDS-polyacrylamide gel electrophoresis and transferred to Optitran nitrocellulose (S and S, Keene, $\mathrm{NH}$, USA), and immunoblotted for Bcl-2 (Dako), BID (R\&D Systems, Minneapolis, MN, USA), cytochrome $c$, caspases 3,8 and 9 (Pharmingen, San, Diego, CA, USA), PARP (Biomol, Plymouth Meeting, PA, USA) and actin (Sigma, St. Louis, MO, USA). Blots were then incubated with horseradish peroxidase-conjugated secondary antibody (Bio-Rad Laboratories, Hercules, CA, USA), washed, and developed by enhanced chemiluminescence (Pierce, Rockford, IL, USA).

\section{Assessment of mitochondrial membrane permeability transition}

Cells were harvested and incubated with $40 \mathrm{nM}$ 3,3-dihexyloxacarbocyanine $\left(\mathrm{D}_{i} \mathrm{OC}_{6}\right.$ Molecular Probes) for $20 \mathrm{~min}$ at room temperature and analyzed by flow cytometry on a Becton Dickinson FACScan. The percentage of cells exhibiting low fluorescence, reflecting loss of inner mitochondrial membrane potential, was determined by comparison with untreated control using CELLQuest software (Becton Dickinson). Alternately, cells were washed and stained by addition of $5,5^{\prime}, 6,6^{\prime}$ - 
tetrachloro-1,1',3,3'-tetraethylbenzimidazolyl carbocyanin iodide (JC1, DePsipher, Trevigen) per the manufacturers instructions, resuspended in PBS and analyzed for loss of red fluorescence.

\section{Assays of caspase activity}

Treated cells were lysed and equivalent quantities of protein assayed per the manufacturers instructions (Caspase-3/CPP32 and FLICE/ Caspase-8 Colorimetric Assay Kits, BioVision). Fold increase in activity was calculated by comparing treated and inhibited samples to untreated control lysates for triplicate measurements for each independent experiment.

\section{Measurement of peroxide radical generation}

Cells were treated with $500 \mathrm{nM}$ 6-carboxy-2',7'-dichlorodihydrodfluorescein diacetate, di(acetoxymethyl ester) (CDCFDA) (Molecular Probes) for $2 \mathrm{~h}$ at $37^{\circ} \mathrm{C}$, fluorescence measured by flow cytometry on a FACScan, and analyzed with CELLQuest software.

\section{Cell cycle analysis}

Cells treated for indicated times were fixed in cold $70 \%$ ethanol, pelleted, and resuspended in staining buffer ( $3.8 \mathrm{mM}$ sodium citrate, $0.5 \mathrm{mg} / \mathrm{ml}$ RNase A, $0.01 \mathrm{mg} / \mathrm{ml}$ propidium iodide) and incubated on ice for $4 \mathrm{~h}$. After resuspension in PBS fluorescence data was collected on a Becton Dickinson FACScan and cell cycle distribution determined with ModFit LT (v2.0, Verity Software).

\section{Statistical analysis}

Significant differences were determined using the student's $t$-test.

\section{Acknowledgements}

Supported by awards CA63753 and CA83705 from the $\mathrm{NIH}$, and award LSA 6407-97 from the Leukemia and Lymphoma Society of America

\section{References}

1. Williams GT, Smith CA, Spooncer E, Dexter TM and Taylor DR (1990) Haemopoietic colony stimulating factors promote cell survival by suppressing apoptosis. Nature 343: 76-79

2. Ashkenazi A and Dixit VM (1998) Death receptors: signaling and modulation. Science 281: 1305-1308

3. Barry MA, Behnke CA and Eastman A (1990) Activation of programmed cell death (apoptosis) by cisplatin, other anticancer drugs, toxins and hyperthermia. Biochem. Pharmacol. 40: 2353-2362

4. Hakem R, Hakem A, Duncan GS, Henderson JT, Woo M, Soengas MS, Elia A, de la Pompa JL, Kagi D, Khoo W, Potter J, Yoshida R, Kaufman SA, Lowe SW, Penninger JM and Mak TW (1998) Differential requirement for caspase 9 in apoptotic pathways in vivo. Cell 94: 339-352

5. Enari M, Sakahira H, Yokoyama H, Okawa K, Iwamatsu A and Nagata S (1998) A caspase-activated DNase that degrades DNA during apoptosis, and its inhibitor ICAD. Nature 391: 43-50

6. Earnshaw WC, Martins LM and Kaufmann SH (1999) Mammalian caspases: structure, activation, substrates, and functions during apoptosis. Annu. Rev. Biochem. 68: 383-424

7. Liu X, Li P, Widlak P, Zou H, Luo X, Garrard WT and Wang X (1998) The 40-kDa subunit of DNA fragmentation factor induces DNA fragmentation and chromatin condensation during apoptosis. Proc. Natl. Acad. Sci. U.S.A 95: 8461-8466
8. Srinivasula SM, Ahmad M, Fernandes-Alnemri T, Litwack G and Alnemri ES (1996) Molecular ordering of the Fas-apoptotic pathway: the Fas/APO-1 protease Mch5 is a CrmA-inhibitable protease that activates multiple Ced-3/ICElike cysteine proteases. Proc. Natl. Acad. Sci. U.S.A 93: 14486-14491

9. LiH, Zhu H, Xu CJ and Yuan J (1998) Cleavage of BID by caspase 8 mediates the mitochondrial damage in the Fas pathway of apoptosis. Cell 94: 491-501

10. LuoX, Budihardjo I, Zou H, SlaughterC and WangX (1998) Bid, a Bcl2 interacting protein, mediates cytochrome $c$ release from mitochondria in response to activation of cell surface death receptors. Cell 94: 481-490

11. Li P, Nijhawan D, Budihardjo I, Srinivasula SM, Ahmad M, Alnemri ES and Wang X (1997) Cytochrome $c$ and dATP-dependent formation of Apaf-1/caspase-9 complex initiates an apoptotic protease cascade. Cell 91: 479-489

12. Srinivasula SM, Ahmad M, Fernandes-Alnemri T and Alnemri ES (1998) Autoactivation of procaspase-9 by Apaf-1-mediated oligomerization. Mol. Cell 1: 949-957

13. Sun XM, MacFarlane M, Zhuang J, Wolf BB, Green DR and Cohen GM (1999) Distinct caspase cascades are initiated in receptor-mediated and chemicalinduced apoptosis. J. Biol. Chem. 274: 5053-5060

14. DeAWJr., Mueller-Dieckmann HJ, Schulze-Gahmen U, Worland PJ, SausvilleE and Kim SH (1996) Structural basis for specificity and potency of a flavonoid inhibitor of human CDK2, a cell cycle kinase. Proc. Natl. Acad. Sci. U.S.A 93 $2735-2740$

15. Morgan DO (1997) Cyclin-dependent kinases: engines, clocks, and microprocessors. Annu. Rev. Cell Dev. Biol. 13: 261-291

16. Carlson BA, Dubay MM, Sausville EA, Brizuela L and Worland PJ (1996) Flavopiridol induces $\mathrm{G} 1$ arrest with inhibition of cyclin- dependent kinase (CDK) 2 and CDK4 in human breast carcinoma cells. Cancer Res. 56: 2973-2978

17. Parker BW, Kaur G, Nieves-Neira W, Taimi M, Kohlhagen G, Shimizu T, Losiewicz MD, Pommier Y, Sausville EA and Senderowicz AM (1998) Early induction of apoptosis in hematopoietic cell lines after exposure to flavopiridol. Blood 91: 458-465

18. Byrd JC, Shinn C, Waselenko JK, Fuchs EJ, Lehman TA, Nguyen PL, Flinn IW, Diehl LF, Sausville E and Grever MR (1998) Flavopiridol induces apoptosis in chronic lymphocytic leukemia cells via activation of caspase-3 without evidence of bcl-2 modulation or dependence on functional p53. Blood 92: 3804-3816

19. Konig A, Schwartz GK, Mohammad RM, Al-Katib A and Gabrilove JL (1997) The novel cyclin-dependent kinase inhibitor flavopiridol downregulates Bcl-2 and induces growth arrest and apoptosis in chronic B-cell leukemia lines. Blood 90 : 4307-4312

20. Senderowicz AM (1999) Flavopiridol: the first cyclin-dependent kinase inhibitor in human clinical trials. Invest New Drugs 17: 313-320

21. Senderowicz AM and SausvilleEA (2000) Preclinical and clinical development of cyclin-dependent kinase modulators. J. Natl. Cancer Inst. 92: 376-387

22. Carlson B, Lahusen T, Singh S, Loaiza-Perez A, Worland PJ, Pestell R, Albanese C, Sausville EA and Senderowicz AM (1999) Down-regulation of cyclin D1 by transcriptional repression in MCF-7 human breast carcinoma cells induced by flavopiridol. Cancer Res. 59: 4634-4641

23. Kitada S, Zapata JM, Andreeff M and Reed JC (2000) Protein kinase inhibitors flavopiridol and 7-hydroxy-staurosporine down-regulate antiapoptosis proteins in B-cell chronic lymphocytic leukemia. Blood 96: 393-397

24. Huang DC, Hahne M, Schroeter M, Frei K, Fontana A, Villunger A, Newton K, Tschopp J and Strasser A (1999) Activation of Fas by FasL induces apoptosis by a mechanism that cannot be blocked by Bcl-2 or Bcl-x(L). Proc. Natl. Acad. Sci. U.S.A 96: $14871-14876$

25. Achenbach TV, Muller R and Slater EP (2000) Bcl-2 independence of flavopiridol-induced apoptosis. Mitochondrial depolarization in the absence of cytochrome c release. J. Biol. Chem. 275: 32089-32097

26. Park DS, Morris EJ, Bremner R, Keramaris E, Padmanabhan J, Rosenbaum M, Shelanski ML, Geller HM and Greene LA (2000) Involvement of retinoblastoma family members and E2F/DP complexes in the death of neurons evoked by DNA damage. J. Neurosci. 20: 3104-3114

27. Shapiro Gl, Koestner DA, Matranga CB and Rollins BJ (1999) Flavopiridol induces cell cycle arrest and p53-independent apoptosis in non-small cell lung cancer cell lines. Clin. Cancer Res. 5: 2925-2938

28. Yang J, Liu X, BhallaK, Kim CN, Ibrado AM, Cai J, Peng TI, Jones DP and Wang X (1997) Prevention of apoptosis by Bcl-2: release of cytochrome $\mathrm{C}$ from mitochondria blocked. Science 275: 1129-1132 
29. Bossy-WetzelE, NewmeyerDD and Green DR (1998) Mitochondrial cytochrome c release in apoptosis occurs upstream of DEVD-specific caspase activation and independently of mitochondrial transmembrane depolarization. EMBO J. 17: $37-49$

30. Gross A, Yin XM, Wang K, Wei MC, Jockel J, Milliman C, Erdjument-Bromage H, Tempst $P$ and Korsmeyer SJ (1999) Caspase cleaved BID targets mitochondria and is required for cytochrome crelease, while $B C L-X L$ prevents this release but not tumor necrosis factor-R1/Fas death. J. Biol. Chem. 274: 1156-1163

31. Wang K, Yin XM, Chao DT, Milliman CL and Korsmeyer SJ (1996) BID: a novel BH3 domain-only death agonist. Genes Dev. 10: 2859-2869

32. Zhou Q, Snipas S, Orth K, Muzio M, Dixit VM and Salvesen GS (1997) Target protease specificity of the viral serpin CrmA. Analysis of five caspases. J. Biol. Chem. 272: 7797-7800

33. Royall JA and Ischiropoulos H (1993) Evaluation of 2', $7^{\prime}$-dichlorofluorescin and dihydrorhodamine 123 as fluorescent probes for intracellular $\mathrm{H} 2 \mathrm{O} 2$ in cultured endothelial cells. Arch. Biochem. Biophys. 302: 348-355

34. Patel V, Senderowicz AM, Pinto D, Jr., Igishi T, Raffeld M, Quintanilla-Martinez L, Ensley JF, Sausville EA and Gutkind JS (1998) Flavopiridol, a novel cyclindependent kinase inhibitor, suppresses the growth of head and neck squamous cell carcinomas by inducing apoptosis. J. Clin. Invest 102: 1674-1681

35. Sherr CJ (2000) The Pezcoller lecture: cancer cell cycles revisited. Cancer Res. 60: $3689-3695$

36. Susin SA, Zamzami N, Castedo M, Hirsch T, Marchetti P, Macho A, Daugas E Geuskens M and Kroemer G (1996) Bcl-2 inhibits the mitochondrial release of an apoptogenic protease. J. Exp. Med. 184: 1331-1341

37. Muzio M, Chinnaiyan AM, Kischkel FC, O'Rourke K, Shevchenko A, Ni J, Scaffidi C, Bretz JD, Zhang M, Gentz R, Mann M, Krammer PH, Peter ME and Dixit VM (1996) FLICE, a novel FADD-homologous ICE/CED-3-like protease, is recruited to the CD95 (Fas/APO-1) death - inducing signaling complex. Cell 85: 817-827

38. Boldin MP, Goncharov TM, Goltsev YV and Wallach D (1996) Involvement of $\mathrm{MACH}$, a novel MORT1/FADD-interacting protease, in Fas/APO-1- and TNF receptor-induced cell death. Cell 85: 803-815

39. Miura M, Friedlander RM and Yuan J (1995) Tumor necrosis factor-induced apoptosis is mediated by a CrmA-sensitive cell death pathway. Proc. Natl. Acad. Sci. U.S.A 92: $8318-8322$

40. Tewari M and Dixit VM (1995) Fas- and tumor necrosis factor-induced apoptosis is inhibited by the poxvirus crmA gene product. J. Biol. Chem. 270: 3255-3260

41. Talanian RV, Yang X, Turbov J, Seth P, Ghayur T, Casiano CA, Orth K and Froelich CJ (1997) Granule-mediated killing: pathways for granzyme B-initiated apoptosis. J. Exp. Med. 186: 1323-1331
42. Friesen C, Herr I, Krammer PH and Debatin KM (1996) Involvement of the CD95 (APO-1/FAS) receptor/ligand system in drug-induced apoptosis in leukemia cells. Nat. Med. 2: $574-577$

43. Landowski TH, Shain KH, Oshiro MM, Buyuksal I, Painter JS and Dalton WS (1999) Myeloma cells selected for resistance to CD95-mediated apoptosis are not cross-resistant to cytotoxic drugs: evidence for independent mechanisms of caspase activation. Blood 94: 265-274

44. Kluck RM, Bossy-Wetzel E, Green DR and Newmeyer DD (1997) The release of cytochrome c from mitochondria: a primary site for $\mathrm{Bcl}-2$ regulation of apoptosis. Science 275: $1132-1136$

45. Marchetti P, Hirsch T, Zamzami N, Castedo M, Decaudin D, Susin SA, Masse B and Kroemer G (1996) Mitochondrial permeability transition triggers lymphocyte apoptosis. J. Immunol. 157: 4830-4836

46. Tang DG, Li L, Zhu Z and JoshiB (1998) Apoptosis in the absence of cytochrome c accumulation in the cytosol. Biochem. Biophys. Res. Commun. 242:380-384

47. Finucane DM, Waterhouse NJ, Amarante-Mendes GP, Cotter TG and Green DR (1999) Collapse of the inner mitochondrial transmembrane potential is not required for apoptosis of HL60 cells. Exp. Cell Res. 251: 166-174

48. Vander Heiden MG and Thompson CB (1999) Bcl-2 proteins: regulators of apoptosis or of mitochondrial homeostasis? Nat. Cell Biol. 1: E209-E216

49. Hedley DW and McCulloch EA (1996) Generation of reactive oxygen intermediates after treatment of blasts of acute myeloblastic leukemia with cytosine arabinoside: role of bcl-2. Leukemia 10: 1143-1149

50. Backway KL, McCulloch EA, Chow S and Hedley DW (1997) Relationships between the mitochondrial permeability transition and oxidative stress during ara-C toxicity. Cancer Res. 57: 2446-2451

51. SchulerM, Bossy-WetzelE, Goldstein JC, Fitzgerald P and Green DR (2000) p53 induces apoptosis by caspase activation through mitochondrial cytochrome $\mathrm{C}$ release. J. Biol. Chem. 275: 7337-7342

52. Wang S, Vrana JA, Bartimole TM, Freemerman AJ, Jarvis WD, Kramer LB, Krystal G, Dent P and Grant S (1997) Agents that down-regulate or inhibit protein kinase $\mathrm{C}$ circumvent resistance to 1-beta-D-arabinofuranosylcytosine-induced apoptosis in human leukemia cells that overexpress Bcl-2. Mol. Pharmacol. 52: $1000-1009$

53. Sundstrom C and Nilsson K (1976) Establishment and characterization of a human histiocytic lymphoma cell line (U-937). Int. J. Cancer 17: 565-577

54. Single B, Leist M and Nicotera P (1998) Simultaneous release of adenylate kinase and cytochrome $c$ in cell death [letter]. Cell Death. Differ. 5: 1001-1003 